Abstract A comprehensive overview is presented about optical fiber-based tunable photonic delay lines, which have been steadily developed over the last decade for the realization of all-optically controlled timing functions. The most widely used techniques, such as those based on slow \& fast light and wavelength conversion associated to dispersion, are described and their physical limitations are discussed in terms of the maximal achievable delay, the associated signal distortion and signal bandwidth. Besides, an entirely different approach for all-optical signal delaying is introduced. This technique is based on movable grating reflectors dynamically generated in highly birefringent optical fibers. This type of delay line has experimentally demonstrated large tunable delaying with a moderate signal distortion for high capacity optical data streams and even for wideband analog signals.

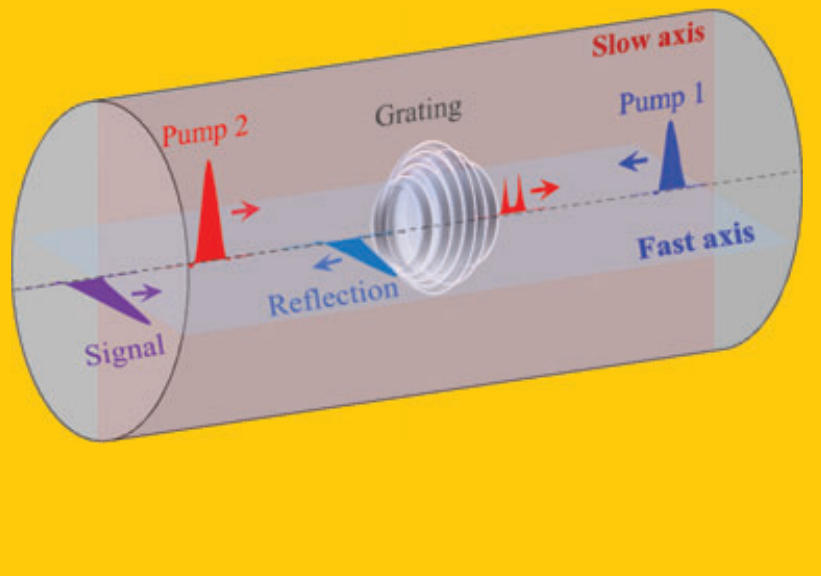

\title{
Tunable photonic delay lines in optical fibers
}

\author{
Sanghoon Chin and Luc Thévenaz
}

\section{Introduction}

The dynamic temporal control of optical signal delaying has been a recent scientific challenge of stimulating interest in the photonic community, since it is expected to be a key requirement for the realization of high capacity networks and their associated modern technologies such as all-optical signal processing and optical buffering in modern optical communication systems $[1,2]$. In addition, such optical tunable delay lines also play a very important role in the domain of microwave photonics, specifically for the generation of arbitrary microwave waveforms, the development of dynamically reconfigurable microwave photonic filters and optical beam forming to feed phase arrayed antennas in radar systems $[3,4]$. They even turn out to be implemented for medical applications, more specifically in optical coherence tomography [5]. During the last decade, many successful experiments on optical signal delaying have been reported and have clearly demonstrated continuous temporal delaying from femtoseconds to several microseconds, using quantum effects in atomic systems [6-12], semiconductor materials [13-19], waveguide properties in specific structures [20-29] and nonlinear optical interactions in optical fibers [30-50]. However, it is interesting to point out that the vast majority of currently available photonic delaying systems can be broadly categorized in two major classes of technique: slow \& fast light and dispersive delay line consisting of the combination of wavelength conversion and group velocity dispersion.

To date, the slow light technique has proved to be a highly flexible timing tool that can provide continuously tunable temporal delays for broadband optical signals. However, it has been also clearly identified that slow light sys- tems - that all acquires a strong dispersion - suffer from a practical limitation in terms of optical delaying capacity. The maximum achievable time delay is strictly bounded by the bandwidth of the system and the delay-bandwidth product is practically of the order of unity. Concretely light pulses cannot experience delays by slow light exceeding a few pulse widths, as a result of the signal distortion associated to the spectral clipping and the higher order dispersion inherent to such systems $[17,51,52]$. This leads to the following conclusion, now widely accepted by the community, that slow light can be considered as an insufficient delaying method for digital optical buffering for future all-optical routers requiring at least 1000-bit delays, even though it was the primary motivation to develop slow light systems. However, optical buffering is just one aspect of slow light and other applications have been recently identified. Slow light can be implemented as a robust solution for retiming multiple channels in digital communication, since it requires only a 1-bit error-free delay. For instance, in a wavelengthdivision multiplexing transmission, a light signal in a given channel can be independently delayed, so that this channel can be precisely re-synchronized to the other with a single bit delaying [53-55]. Moreover, when analogue signals are involved in communication systems, slow light can offer the possibility to produce tunable delays over more than one signal period - or equivalently to generate a $2 \pi$ phase shift on the carrier - for an efficient processing of microwave signals for instance [56-58].

As an efficient solution for all-optically buffering multibit sequences in high speed networks, dispersive delay lines seem to be particularly promising, since this technique has already shown its potential capability to produce more than 1000 -bits delays for $10 \mathrm{Gbit} / \mathrm{s}$ transmissions, with clear pos- 
sibilities for even faster data rates [30,33]. However, it must be mentioned that this technique also suffers from an inevitable signal distortion, observed as pulse broadening, since a large group velocity dispersion (GVD) is essential to generate such long time delays. This results in a trade-off similar to slow light between system bandwidth and delaying power. However, the GVD-induced frequency chirp, which is the basic mechanism giving rise to temporal broadening, can be compensated by simply imposing an extrinsic frequency chirp across the pulse with opposite sign. It has been even experimentally demonstrated that signal pulses could experience ultra large delays with nearly zero-broadening effect while the GVD-induced frequency chirp was entirely compensated using optical phase conjugation [30,33]. Nevertheless, the generation of large amount of delays preserving a high fidelity of the optical information remains a scientific challenge yet.

In this paper, we shall briefly review the state-of-the-art of these two techniques and address their working principle. Then we shall present some recent experimental results on optical signal delaying based on a novel architecture recently developed by our group. This entirely different class of all-optical delay line, compared to the two above mentioned techniques, makes use of dynamic Brillouin grating reflectors in polarization maintaining optical fibers. We have experimentally demonstrated extensive temporal delaying for broadband optical signals, inflating the delay-bandwidth product by several orders of magnitude.

\section{Slow \& fast light in optical fibers}

Slow and fast light refers to a technique modifying in a given optical medium the group velocity $v_{g}$ at which a light signal is travelling to make it substantially different from the normal group velocity in this medium. In principle, when a pulsed signal propagates through a dispersive medium, the motion of the pulse envelope is governed by the group velocity since signal pulses can be decomposed into a set of monochromatic waves spanning over a frequency interval, so each propagating at a different velocity. The group velocity $v_{g}$ depends directly on the dispersion in the material and can be expressed as $v_{g}=c / n_{g}$, where

$$
n_{g}=n+\omega \frac{d n}{d \omega} .
$$

with $n$ the refractive index in the medium, classically determined by the ratio between the light velocities in vacuum and in the given optical medium.

So, the pulse can propagate through the dispersive medium with a reduced or increased velocity when compared to the phase velocity $c / n$, depending on the dispersion characteristics of the material $d n / d \omega$. In general, a steep linear variation of refractive index $n$ with respect to optical frequency $\omega$ is observed within spectral regions where sharp spectral resonances are present. The induced dispersion can then be calculated using Kramers-Kronig relations, as shown in Fig. 1. An anomalous dispersion $(d n / d \omega<0)$,

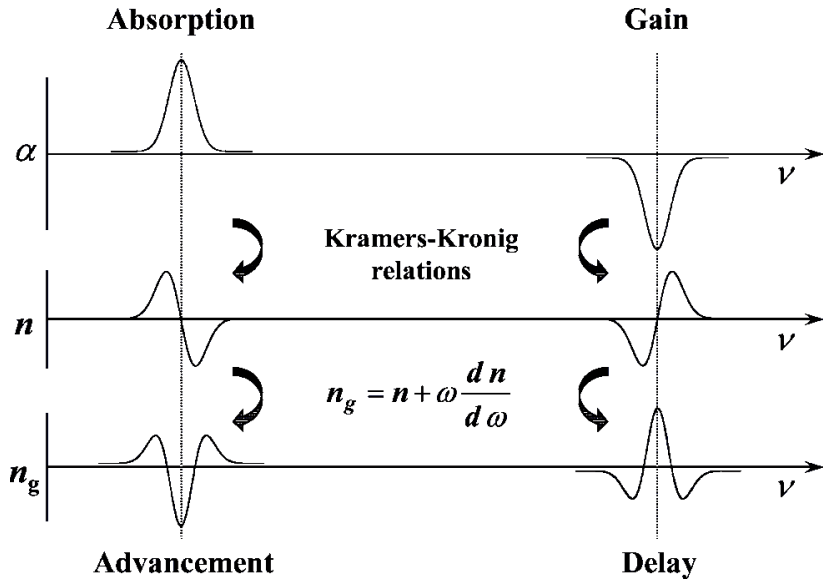

Figure 1 Sharp spectral changes in the optical transmission (top) within spectral resonances (either absorption or gain bands) leads to refractive index variations with respect to optical frequency (middle), which can be calculated using Kramers-Kronig relations. The dispersion appearing in the resonance leads to a change in group velocity (bottom), namely the generation of slow and fast light.

as created in the center of an absorption line, leads to a decrease in group index and thus a higher speed of the pulse envelope, hence resulting in a temporal advancement for a signal pulse or fast light. On the contrary, a gain line will induce a normal dispersion $(d n / d \omega>0)$ in the material, resulting in an increase in group index making the light pulse slow down, hence generating a pulse delay or slow light.

As a result, the control of the signal velocity requires finding the specific physical processes that can induce optical resonances with the proper spectral properties in the material. Over the last decade, a flurry of solutions for tailoring dispersive properties in optical media has been actively developed in diverse materials, and astonishing controls of group velocity were experimentally demonstrated using a vast choice of physical mechanisms. Early experiments on slow light propagation were first conducted using atomic resonances in atomic gases, then later in crystalline solids, resulting in a remarkable reduction of the group velocity [6-12]. However, these approaches were still seen like far from acceptable for real applications since they show no flexibility in the operating wavelength and the ultra-narrow bandwidth of the spectral hole has restricted the signal bandwidth to the $\mathrm{kHz}$ range. A significant step towards real application was then accomplished when slow and fast light has been achieved in optical fibers, using optical interactions generated by nonlinear optical phenomena. In most nonlinear processes, energy transfer occurs from one optical wave to another wave, mediated by an idler wave once a required phase matching condition is satisfied. If a signal pulse benefits from the energy transfer in the process to experience a linear gain, the associated nonlinear phase shift will induce a normal dispersion that leads to slow light and the propagation speed of the signal will be reduced through the medium. On the other hand, an optical wave that undergoes a linear loss from the process will see its sig- 
nal envelope accelerated, so that fast light will be observed in the transmission.

To engineer dispersion characteristics in optical fibers, various physical phenomena have been utilized over the last few years, such as stimulated Brillouin $[42,44]$ and stimulated Raman [46] scatterings, narrow-band optical parametric amplification [40] and coherent population oscillation $[41,59]$. However, it turns out that stimulated Brillouin scattering in fibers is the most efficient nonlinear mechanism for the generation of slow light due to its high intrinsic gain and its large flexibility for spectral tailoring at any operating wavelength. To date, Brillouin slow light has already shown the possibilities to manipulate the group velocity of a light signal from as slow as $\mathrm{v}_{g}=71000 \mathrm{~km} / \mathrm{s}$ to superluminal propagation, even reaching negative velocities [43]. In the following sub-sections, we will make an overall review on Brillouin slow light in fibers, with a special focus on actual limitations for real applications and on proposed solutions to overcome these issues. A more complete review showing the most fascinating experimental results on signal pulse delaying in Brillouin slow light can be found in [48].

\subsection{Principle and limitations of Brillouin slow light}

Stimulated Brillouin scattering in optical fibers is described as a nonlinear interaction between two counter-propagating optical waves (a strong pump at $v_{\text {pump }}$ and a weak probe at $\left.v_{\text {probe }}\right)$ in which their mutual coupling during propagation in fibers is mediated by an acoustic wave. When a specific phase matching condition is realized, that is, the spectral separation between the two interacting waves $\left(\Delta v=v_{\text {pump }}-v_{\text {probe }}\right)$ is identical to the acoustic wave frequency $v_{B}\left(v_{B}\right.$ called the Brillouin frequency shift), the beating signal resulting from the interference between the two optical waves reinforces the acoustic wave through the effect of electrostriction. Incidentally, the material density is periodically modulated along the fiber by the acoustic pressure wave through the elasto-optic effect, leading to a periodic travelling modulation of the refractive index $[60,61]$. As a result, the acoustic wave generated in the fiber acts as a moving grating reflector, showing the exact pitch to backscatter the interacting waves. The net effect of the waves scattering results in transferring the energy from the higher frequency wave (the pump) to the lower frequency wave (the probe), the scattered light undergoing a Doppler shift due to the grating motion. So, the probe wave experiences an exponential growth, referred to as SBS amplification, as shown in Fig. 2. On the contrary, the pump wave experiences an exponential attenuation since a part of its optical energy is transferred to the probe wave. Therefore, SBS can also be assimilated to a loss process as far as the pump is concerned.

Fig. 3 depicts the schematic principle to generate slow and fast light using stimulated Brillouin scattering (SBS) in optical fibers. A basic Brillouin slow light system consists of a very simple configuration, in which a continuous-wave (CW) pump is launched into one end of a single mode optical fiber (Brillouin shift $v_{B}$ ), while a signal is simultaneously
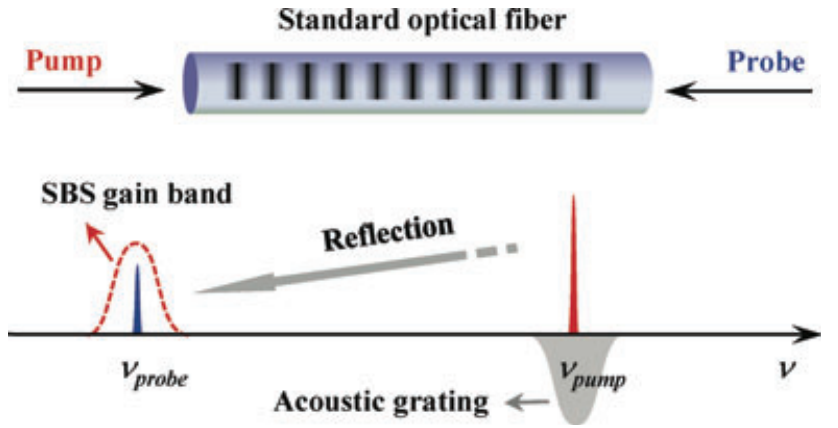

Figure 2 (online color at: www.lpr-journal.org) General description of the ordinary stimulated Brillouin scattering process in standard optical fibers, generating Brillouin gain and loss resonance that are spectrally centered at the probe and pump frequencies, respectively.

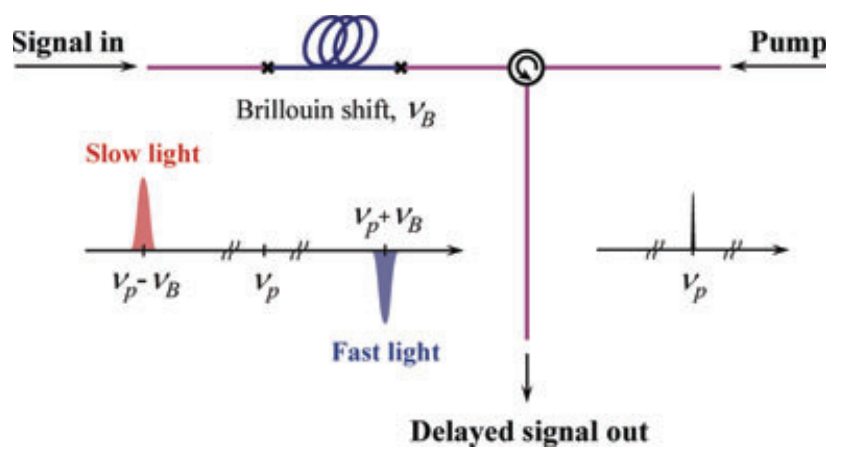

Figure 3 (online color at: www.lpr-journal.org) Schematic diagram to generate slow and fast light based on stimulated Brillouin scattering in optical fibers.

introduced into the other end of the fiber. When the pump propagates through the fiber, acoustic phonons are created by electrostriction via the interference with the signal and diffract photons from the pump light to the signal wave, thus producing a gain resonance for the signal at frequency $-v_{B}$ below the pump frequency. The spectral width of the Brillouin resonance is typically as narrow as $30 \mathrm{MHz}$ due to the slow decay of the acoustic wave. Similarly, besides the gain resonance, the pump creates a SBS loss resonance with an identical bandwidth at frequency $+v_{B}$ above the pump frequency. Therefore, when the signal is spectrally placed at the center of the gain or loss resonance (resulting from the particular phase matching conditions of the SBS process) the group velocity of the signal can be efficiently reduced or increased, respectively. However, the inherently narrow spectral width of Brillouin resonances raises a major critical limitation in Brillouin slow light system, since it practically limits the signal data rate to some $50 \mathrm{Mbit} / \mathrm{s}$. So, a solution is required to match the real multi-Gbit/s data rates in communication systems.

In absence of gain saturation or pump depletion in the SBS process, the time delay via Brillouin slow light can be expressed by the simple relation $[48,62]$ :

$$
\Delta T_{d}=\frac{L}{c} \Delta n_{g} \simeq \frac{G}{2 \pi \Delta v_{B}},
$$


where $G=g_{o} I_{p} L$ is the logarithmic net Brillouin gain through the entire fiber length $L\left(g_{o}\right.$ : linear Brillouin gain coefficient in $\mathrm{m} / \mathrm{W}), I_{p}$ is the pump intensity in $\mathrm{W} / \mathrm{m}^{2}$ and $\Delta v_{B}$ is the full with at half maximum of the Brillouin resonance in $\mathrm{Hz}$. Therefore, a signal pulse can be continuously delayed or accelerated in Brillouin slow light systems, by simply varying the optical power of the pump. However, a second limitation in Brillouin slow light can be seen at this stage. In Eq.(2), the relative time delay $\Delta T_{d}$ has a logarithmic dependence on the net signal gain in most implementations of Brillouin slow light, since $\mathrm{e}^{G}$ represents the net signal gain. The significant amplitude change accompanying a large delay (showing a linear relation of $1 \mathrm{~ns}$ of delay per $\mathrm{dB}$ of gain in native Brillouin slow light systems) can be considered as a serious impairment since the strong amplification of the signal significantly changes the power budget in a real communication system.

Finally, the effect of the interaction on the signal can be described as a linear time-invariant system and therefore represented by a linear spectral transfer function [63]. A Brillouin slow light system corresponds to a linear low-pass system and, as a result of the bandwidth clipping, the signal may undergo a strong distortion observed as a temporal broadening of a pulse signal. This lead to another limitation in the system in terms of the maximum achievable time delay supporting an acceptable signal distortion, since the intersymbol interference due to pulse broadening will degrade the signal fidelity. In practice the delay-bandwidth product of linear slow light systems is close to unity, so that a pulse cannot be delayed significantly more than its temporal width.

\subsection{Broadband Brillouin slow light}

As a consequence of the linear dependence of the Brillouin gain on pump power, when a polychromatic wave is used as a pump in the SBS process, each monochromatic wave composing the pump spectrum will generate its own Brillouin gain resonance at its specific central frequency, given by the frequency matching condition for stimulated Brillouin scattering $[64,65]$. Then the effective Brillouin gain spectrum is a combination of each gain spectrum generated by each spectral component of the polychromatic pump wave. As a result, the spectral profile of the effective Brillouin resonance can be drastically engineered and shaped by simply modifying the pump power spectrum. This way the bandwidth of Brillouin slow light can be made arbitrarily large by actively broadening the pump power spectrum. The power spectrum of the effective SBS gain $g_{e f f}(v)$ is expressed by the convolution of the pump power spectrum and the intrinsic Brillouin gain spectrum $g_{B}(v)[37,66]$ :

$$
g_{\text {eff }}(v)=P(v) \otimes g_{B}(v)
$$

where $\otimes$ denotes the convolution product and $P(v)$ is the normalized pump spectral density (integration over spectrum is unity). For a Lorentzian shape of the pump spectrum, the effective gain curve remains Lorentzian and its width is given by the sum of the two spectral widths $\Delta v_{B}+\Delta v_{P}$, $\Delta v_{B}$ being the spectral width of the natural Brillouin gain and $\Delta v_{P}$ being that of the pump power.

When a broadband noise is directly superposed to the injection current of a pumping semiconductor laser, the power spectrum of the pump can be significantly broadened and its spectral width can be much larger than that of the natural Brillouin resonance $\left(\Delta v_{P} \gg \Delta v_{B}\right)$. This way, the spectral shape of the effective Brillouin gain turns out to be essentially identical to the pump power spectrum, the contribution from the natural linewidth being considered as approximately monochromatic in the convolution of Equ.3. Actually, the enlargement of the effective SBS bandwidth has removed a major deadlock for the implementation of Brillouin slow light in real applications, making Brillouin slow light a suitable delaying system for multi-Gbits/s transmissions. However, it must be pointed out that this bandwidth extension requires a quadratic increase of the pump power to maintain an equivalent delay. This results from the combination of two causes: first, the peak value of the effective Brillouin gain decreases proportionally to the relative spectral broadening, at constant total power. Secondly, as shown in Eq.(2), the amount of time delay is inversely proportional to the spectral width of the Brillouin resonance. The 2 effects contribute in the same proportion to result in a quadratic dependence. If the same normalized delay must be maintained (i. e. the delaying in terms of pulse width units is constant), the pump power must be increased proportionally to the bandwidth.

Broadband Brillouin slow light, as depicted in Fig. 4a, was for the first time experimentally demonstrated by Gonzalez-Herráez et al. using a randomly modulated pump source [37]. By directly modulating the current of a pump laser diode, the FWHM bandwidth of the Brillouin resonance could be effectively enlarged up to $325 \mathrm{MHz}$, therefore allowing a $2.7 \mathrm{~ns}$ signal pulse to be continuously delayed up to $3 \mathrm{~ns}$. Then a thorough study on this principle was theoretically and experimentally carried out by Zhu et al. [67], showing that the maximum achievable bandwidth is essentially limited by the spectral overlapping due to the associated SBS loss resonance, as shown in Fig. 4b. The simultaneous broadening of the Brillouin loss resonance actually limits the spread of the Brillouin gain, so that the maximal bandwidth cannot exceed the Brillouin frequency $v_{B}$, which is typically $11 \mathrm{GHz}$. However, Song et al. proposed a solution to further extend the bandwidth of Brillouin slow light by introducing another pump positioned at frequency $+2 v_{B}$ above the first pump frequency [68]. As in any SBS process, these two pumps generate distinct Brillouin gain and loss spectra at $\pm v_{B}$ with respect to their pump frequency. The particular special positioning of the 2 pumps makes the loss spectrum from Pump 1 and the gain spectrum from Pump 2 identical in terms of bandwidth, peak amplitude and central frequency, so that they perfectly overlap and mutually neutralize for a perfect compensation, as shown in Fig. 4c. This clears the spectral region formerly occupied by the loss resonance from any gain or loss and makes possible a further doubling of the pump broadening. This way the Brillouin bandwidth could be enlarged up to 


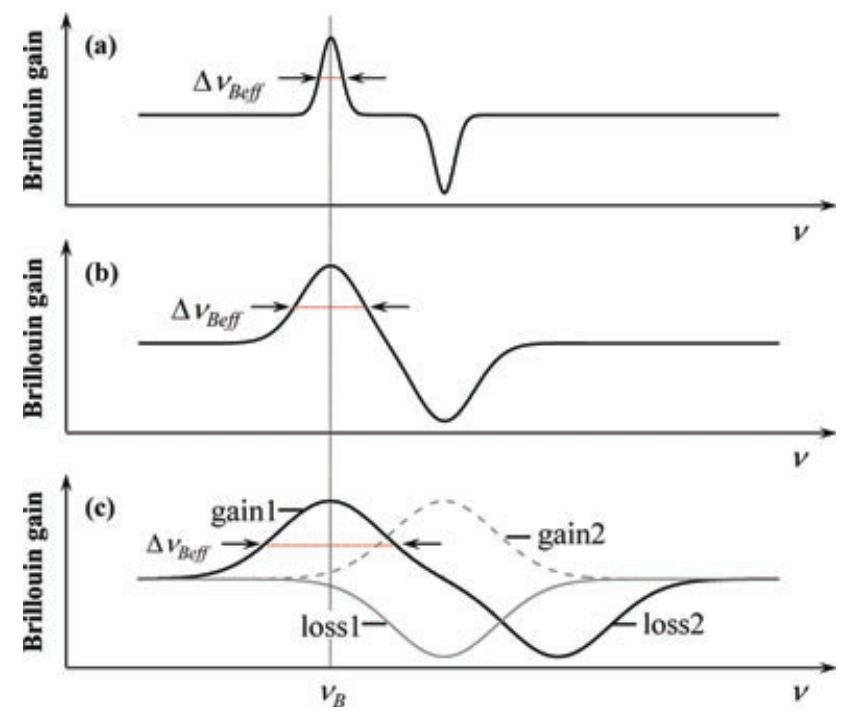

Figure 4 (online color at: www.lpr-journal.org) Effective SBS gain spectra induced by a modulated pump source. (a) The Brillouin spectrum is enlarged by the broadband pump, which simultaneously induces Brillouin gain/loss resonances showing a broadened bandwidth. (b) The maximum achievable SBS bandwidth is limited by the overlap of the SBS gain/loss resonances. The right wing of the gain spectrum and the symmetric left wing of the loss mutually cancel, resulting in a maximum bandwidth of $\Delta v_{B} \sim v_{B}$. (c) However, when judiciously introducing another pump (Pump 2) the SBS gain 2 generated by the Pump 2 compensates the SBS loss 1 , which allows a further extension of the effective bandwidth of the SBS gain 1.

$25 \mathrm{GHz}$ and a $37 \mathrm{ps}$ pulse was successfully continuously delayed up to a $30 \%$ fractional delay [68].

This direct modulation technique, while removing the bandwidth deadlock in a transmission channel, is still seeking for a solution for the implementation in wavelength division multiplexed optical systems. Delays can be controlled independently and simultaneously for all operating channels using Brillouin slow light, but the number of broadband coherent pump sources and associated electrical components required for an independent control of each channel turns out to be a real issue. To overcome it, an alternative approach to synthesize broadband Brillouin slow light was proposed using incoherent light sources to generate multiple Brillouin pump waves, i. e. sources based on amplified spontaneous emission (ASE) [55]. A broadband ASE light is sent into a Fabry-Perot filter and the periodic lines present in the spectral transmission of the filter are used as multiple Brillouin pumps. In this configuration, the operation frequency and the spectral width of the pumps can be tunable, by simply varying the transmission characteristics of the filter.

\subsection{Transparent Brillouin slow light}

The exponential growth of the signal power accompanying the temporal delaying effect is inherent to any Brillouin slow light system, as expressed in Eq.(2), since large dispersion

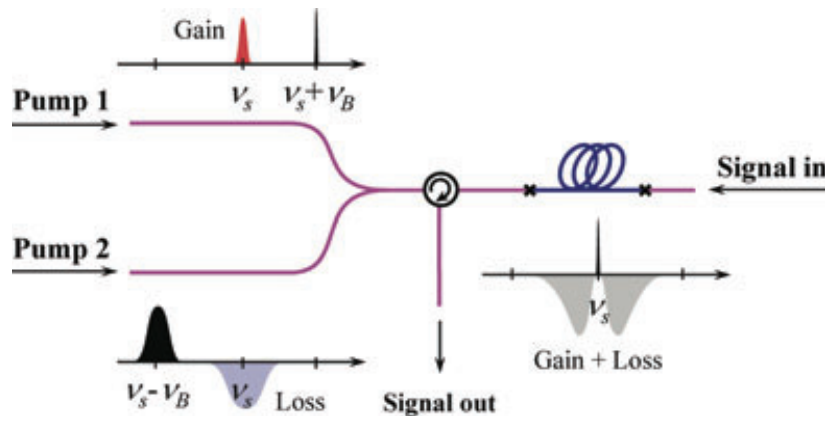

Figure 5 (online color at: www.lpr-journal.org) Principle for the generation of zero-gain SBS spectral resonances. Two distinct Brillouin pumps produce Brillouin gain and loss resonances with different spectral widths centered at the signal frequency, resulting in a narrowband transparency window for the signal.

and gain are intimately associated in a spectral resonance. Chin et al. could, however, find a solution to experimentally demonstrate tunable time delays through Brillouin slow light with minor amplitude change. Figure 5 shows the schematic diagram to realize zero-gain Brillouin slow light propagation. Pump 1 at frequency $+v_{B}$ above the signal frequency generates a Brillouin gain resonance at the central frequency of the signal pulse with a linear gain $G_{1}$ and a bandwidth $\Delta v_{1}$. Pump 2 at frequency $-v_{B}$ below the signal frequency creates a loss of equal strength $-G_{1}$ at the same frequency. However, the spectrum of Pump 2 was broadened, so that the bandwidth of the loss spectrum $\Delta v_{2}$ is substantially larger than $\Delta v_{1}\left(\Delta v_{2} \gg \Delta v_{1}\right)$. In such a situation, a spectral hole is created in the middle of the loss spectrum to open a transparency window at the signal frequency as a result of the complete compensation of gain and loss. This situation is thus fairly similar to the case of slow light systems in solids and gases where the signal is spectrally positioned in the narrow transparency gap between 2 absorptive resonances. On the other hand, resonance-induced dispersions by gain and loss are fairly different and do not compensate, since the resonance bandwidths are differently set (see Equ.2). This way, continuous signal delays up to $12 \mathrm{~ns}$ were obtained for a $50 \mathrm{~ns}$ pulse with less than $1 \mathrm{~dB}$ variation in the signal power over the whole delay range. Using this gain compensation technique, signal advancement or fast light propagation can be also obtained by simply swapping the spectral positions of the two pumps. So, a large bandwidth Brillouin gain resonance is created at the signal frequency, and a narrow bandwidth loss resonance makes a spectral hole burning in the center of the gain resonance. However, it must be mentioned that the extra noise generated by the amplification process is not cancelled by the loss interaction and remains present in the delayed signal.

\subsection{Brillouin slow light with pulse broadening compensation}

In absence of gain saturation or pump depletion, a Brillouin slow light system can be identified to a linear time-invariant 
system and its transfer function $T(\omega)$ can be expressed as $[62,69]$ :

$$
T(\omega)=\exp \left[\frac{G}{2}\left(\frac{1}{\left(1+2 \omega / \Gamma_{B}\right)^{2}}-i \frac{2 \omega / \Gamma_{B}}{\left(1+2 \omega / \Gamma_{B}\right)^{2}}\right)\right] .
$$

Since the transfer function of the Brillouin resonance shows a complex response, the amplitude and the phase of the signal are transformed by the real and the imaginary parts in the exponent of $T(\omega)$, respectively. Actually, the frequency dependence of the amplitude term acts as a low-pass spectral filtering that attenuates the high frequency signal components and distorts its shape. Therefore, a temporal broadening of the pulse signal is usually observed when the signal emerges from the Brillouin delay line, which is broader for larger delays. This is referred as gain broadening, $B_{G}$. On the other hand, due to the large induced dispersion associated to the imaginary part in the exponent of $T(\omega)$, the higher order dispersion terms (the first order term is kept since it is directly responsible of the signal delaying) induces an additional phase distortion, referred as dispersion broadening, $B_{D}$. Therefore, the total pulse broadening, $B_{\text {total }}$ is found by calculating the geometrical sum of the two broadening factors: $B_{\text {total }}^{2}=B_{G}^{2}+B_{D}^{2}$, since they are by essence associated to the real and imaginary parts of the transfer function. It is interesting to mention that the amplitude response $G(\omega)$ is spectrally symmetric and thus contains only even terms in its polynomial expansion, while the phase response is anti-symmetric and contains only odd terms. These two broadening effects can in no way mutually cancel to compensate the signal distortion, since they are distinctly related to purely real and imaginary quantities. Moreover, since there is no quadratic dependence in the phase response, an introduction of ordinary fiber chromatic dispersion in a Brillouin slow light system can in no way bring any compensation and just add further distortion. As a result, larger signal delay is always accompanied by more pulse broadening in any type of linear slow light systems.

Several linear slow light systems were designed to overcome this delay-distortion issue, mostly based on a particular shaping of the spectral resonance. These systems could partially reduce the induced signal distortion while preserving the fractional delay [70-75], but this approach could not fully eliminate the distortion that eventually limits the maximum delay to one pulse width or so. Since this distorted response is intimately related to the delaying process as described by the theory of linear systems, the need to introduce nonlinear elements is unavoidable to realize distortion-less signal delaying in slow light systems $[51,63,76,77]$. In an experiment reported by Wiatrek et al. a distinct saturated Brillouin amplifier was used as a nonlinear system, which could mitigate the broadening effect [78] and result in signal delays up to 1 bit with a minor distortion. However, the time delay per unit gain is reduced by the saturated amplifier since the amplifier provides gain with no real delaying effect.

Chin et al. also proposed a nonlinear configuration to achieve an efficient compensation of signal distortion, by combining a Brillouin amplifier with a fast saturable absorber made of a fiber nonlinear optical loop mirror (NOLM), as shown in Fig. 6 [31]. This configuration comprises two basic building blocks: a Brillouin slow light element and a NOLM as a nonlinear regeneration element. As in a typical Brillouin slow light system, a signal pulse experiences temporal delays and the associated temporal broadening, before entering into the NOLM. In the time domain, the NOLM can be considered as a pulse compressor since it sharpens the shape of the input pulse due to the intensity dependence of the transmission through the NOLM. Therefore, in frequency domain, the signal bandwidth can be fully recovered since the frequencies that have been attenuated by the slow light element can be regenerated as a consequence of the pulse temporal compression. The transmission through the NOLM shows a cubic dependence on the input intensity, so that the compression factor can be expected to be $3^{-1 / 2}$ for signal pulses with a Gaussian intensity profile. This way a signal pulse with duration of $27 \mathrm{~ns}$ was continuously delayed up to $36 \mathrm{~ns}$, equivalent to a fractional delay of 1.3 without any broadening. Besides, using this type of slow light system, the fidelity of the delayed signal was greatly enhanced in terms of signal-to-noise ratio. The contrast between signal "on" and "off" states is substantially improved by the gating effect of the NOLM intensity transmission.

\section{Dispersive delay line in optical fibers}

Another approach for the development of all-optically controlled delay line has been proposed using the combination of wavelength conversion and group velocity dispersion. This delaying technique is in essence entirely different from slow light-based schemes, since this method exploits the natural dispersion of the material and does not require a

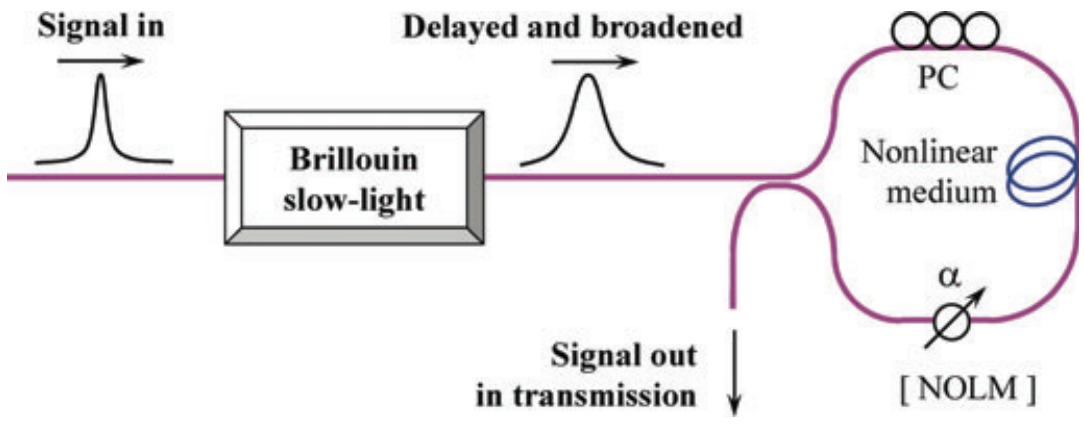

Figure 6 (online color at: www.lprjournal.org) Principle to delay a signal pulse without broadening in a Brillouin slow light system. In this configuration, a nonlinear optical loop mirror (NOLM) acts as a pulse compressor, so that the pulse broadening accompanying the time delaying is completely compensated while fully preserving the temporal delaying power of the Brillouin slow light system. PC; polarization controller, $\alpha$; attenuation factor. 


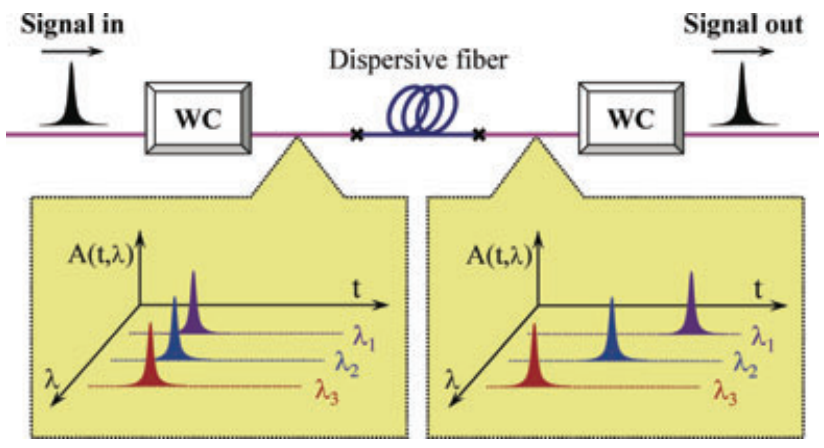

Figure 7 (online color at: www.lpr-journal.org) Schematic diagram of the principle to generate time delays in a dispersive delay line. The system consists in a wavelength conversion followed by the propagation in a highly dispersive optical medium. WC; wavelength convertor.

narrow spectral resonance to modify the dispersive response like in slow light systems.

The propagation velocity of a signal pulse while traveling through a dispersive medium turns out to be wavelengthdependent as a result of the chromatic dispersion or group velocity dispersion (GVD) in the material. So, the transit time of the signal through the medium can be made tunable by simply varying the signal wavelength; this is the simple principle that is used for tunable signal delaying in dispersive delay lines. Figure 7 depicts this principle. In a first stage, the wavelength of a signal pulse is converted into another target wavelength using wavelength conversion. Then the wavelength-converted pulse propagates through an optical medium showing a large overall dispersion, such as long segments of optical fiber [30, 32, 33, 45, 50,79] or a highly chirped fiber Bragg grating [49]. Due to the intrinsic chromatic dispersion of the medium, the incident pulse emerges from the dispersive element with a relative time delay when compared to the transit time as it would be at the original wavelength of the pulse. For a given GVD, the obtained time delay $\Delta T_{d}$ as a function of the signal wavelength change $\Delta \lambda$ can be roughly estimated using a first order approximation, valid for small wavelength change, as:

$$
\Delta T_{d}=D L \Delta \lambda
$$

where $D$ is the GVD coefficient of the dispersive element expressed in units of $\mathrm{ps} /(\mathrm{km} \times \mathrm{nm}), L$ being the length of this element. In a third stage, the delayed wavelength-converted pulse is delivered into a second wavelength convertor, so that the original wavelength of the temporally delayed pulse is restored.

Sharping et al. experimentally demonstrated this concept of dispersive delay line and could generate continuously tunable time delays up to $800 \mathrm{ps}$ for a $10 \mathrm{ps}$ signal pulse [45]. This was already more than one order of magnitude better than the best delaying system based on slow light. In most dispersive delay lines, the wavelength conversion of the signal pulses is realized using optical nonlinear processes such as four wave mixing or massive spectral broadening through self-phase modulation in optical fibers or Si-waveguides.
However, this type of wavelength convertors requires a strong pump power to obtain high conversion efficiency, and the associated excess noise or the spectral broadening resulting from the conversion processes degrades the fidelity to the original signal. Another type of wavelength convertor has been soon proposed, based on periodically poled lithium-niobate waveguide (PPLN) [80]. The PPLN wavelength convertor improves the performance of signal delaying in terms of a reduced additive noise, a faster reconfiguration time and a larger maximum achievable delay since the wavelength tuning range is inherently more extended. Following the same motivation for a more efficient conversion, in a recent work [32] cross gain modulation in a semiconductor optical amplifier has been implemented to efficiently realize the wavelength conversion using a low pump power level and over a broad wavelength range.

Eq.(5) indicates that the GVD is the key quantity fixing the amount of time delay. On the other hand, it leads to signal distortion expressed by pulse broadening since the frequency components forming the signal pulse must temporally disperse. It means that the dispersive delay line is also subject to a trade-off relation between maximum achievable time delay and signal distortion. A larger time delay through dispersive delay line can be achieved by simply increasing the amount of wavelength change. However, in practice, the bandwidth of wavelength conversion is limited to a few tens of nanometers by the different techniques that are currently available. Alternatively, a medium showing a larger value of GVD can be utilized to enhance the achievable time delay. However, in this case, a non-negligible temporal broadening of the signal pulse can accompany the temporal delaying, limiting by essence the system bandwidth. Figure 8 shows the anticipated pulse broadening and the required wavelength shift as a function of GVD, in order to achieve a 1000-bits delay for a 100 ps transform limited Gaussian pulse, representative of a $10 \mathrm{Gbit} / \mathrm{s}$ transmission. Actually, the delaying system can be properly designed using the information in this graph. It is clearly seen that a GVD larger than $1000 \mathrm{ps} / \mathrm{nm}$ is necessarily needed to delay

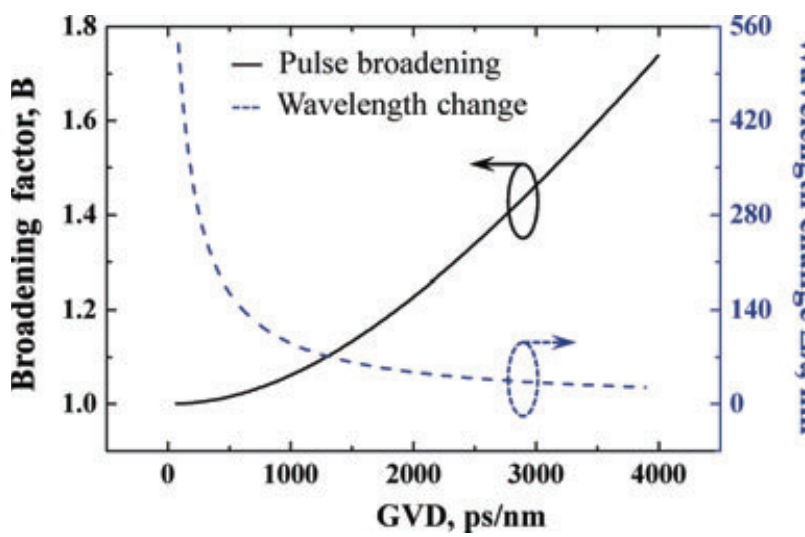

Figure 8 (online color at: www.lpr-journal.org) Associated pulse broadening and the required wavelength change to achieve 1000bits delay for $100 \mathrm{ps}$ transform-limited Gaussian pulse as a function of group velocity dispersion (GVD). 
a signal pulse up to 1000 bits with wavelength shift smaller than $100 \mathrm{~nm}$. For example the GVD of $3600 \mathrm{ps} / \mathrm{nm}$ used in [33] requires a wavelength change of $28 \mathrm{~nm}$, which seems to be a realistic value. However a pulse broadening by a factor of 1.6 will be associated to this delaying.

The pulse broadening could, however, be partially compensated by using a chirped fiber Bragg grating, which has a strong dispersion with opposite sign with respect to the dispersive fiber used as a delay line [80]. However, Y. Okawachi et al. could experimentally demonstrate an excellent solution to minimize the inevitable pulse distortion in dispersive delay line, using temporal phase conjugation [33]. After propagating through a highly dispersive fiber, the signal pulse experienced a relative time delay subject to temporal broadening. Then the optical phase of the pulse was temporally conjugated using four-wave mixing and re-circulated into the same dispersive fiber. As a result, the time delay was doubled and the induced pulse broadening could be fully compensated when exiting from the fiber, since the slope of the dispersion-induced chirp is reversed by phase conjuguation. Using this configuration, a fractional delay of 2430 was achieved for a $10 \mathrm{Gbit} / \mathrm{s}$ NRZ signal while preserving a high signal fidelity. Recently, the maximum delay was amazingly extended to $7.34 \mu$ s by simply cascading multiple discrete delay lines [30].

\section{Movable grating-based optical delay line}

In this section, an entirely different approach for realizing all-optically controlled delay lines will be addressed. The time-of-flight is here simply changed by reflecting the signal on a movable reflector, just like in a classical optomechanical delay line. The delay is therefore given by the round-trip time along the optical path that can be modified by the physical displacement of the reflector. The technique here described offers the possibility to dynamically generate reflectors at any position along an optical fiber through the intercession of a purely optical interaction. The dynamic generation of reflectors is based on the process of ordinary stimulated Brillouin scattering through which optically-generated acousto-optic gratings can be localized at any desired position along an optical fiber. If this fiber is highly birefringent, it turns out that a signal propagating along the orthogonal birefringence axis will be reflected at a wavelength clearly separated from the signals writing the grating. The functions of reflector generation and signal reflection can therefore be realized with totally distinct optical signals.

\subsection{Generation of dynamic Brillouin gratings}

The classic SBS interaction of two intense waves, here called "pumps" for the sake of simplicity, (Pump 1 at $v_{P 1}$ and Pump 2 at $v_{P 2}$, and $v_{B}=v_{P 1}-v_{P 2}$ ) is used to create the acoustic grating while these two pumps propagate along one polarization eigenaxis of the fiber. In this case, the longitudinal nature of the acoustic wave enables an indistinct and equal reflection of light for all states of polarization. Actually, a light signal polarized along the orthogonal polarization axis with respect to the pumps will be equally reflected, but at a distinct optical frequency $v_{S}$ since the birefringence modifies the optical frequency satisfying the Bragg condition [81]. It turns out that the functionality of this grating is analogous to a weak Bragg grating written in an optical fiber, so that the spectral bandwidth and the reflectance of the grating can be dynamically controlled by simply changing the amplitude and the time dependence of the two pump waves [82]. For this reason, such a Bragg reflector is denominated dynamic Brillouin grating (DBG). DBGs have been already implemented as a promising solution for the development of optical fiber sensors [83-85] and optical signal processing [34,86-88]. The key advantage of DBG is that the creation of the Brillouin grating and its interrogation can be realized using two uncorrelated processes, based on distinct optical waves.

Figure 9 depicts the configuration in optical frequencies and states of polarization for the two pumps and the signal waves to generate a dynamic grating in a polarizationmaintaining fiber. Due to the birefringence of such a fiber the Bragg condition can be met at two different optical frequencies for the orthogonal polarization eigenmodes, since the polarization-dependent refractive index leads to a different optical pitch of the grating for the 2 polarizations, the physical pitch being isotropically fixed by the acoustic wavelength. Therefore, the central frequency of the dynamic grating can be simply calculated by the expression [81]:

$$
\Delta v=v_{S}-v_{P 1}=\frac{\Delta n_{g}}{n_{y}} v_{P 1}
$$
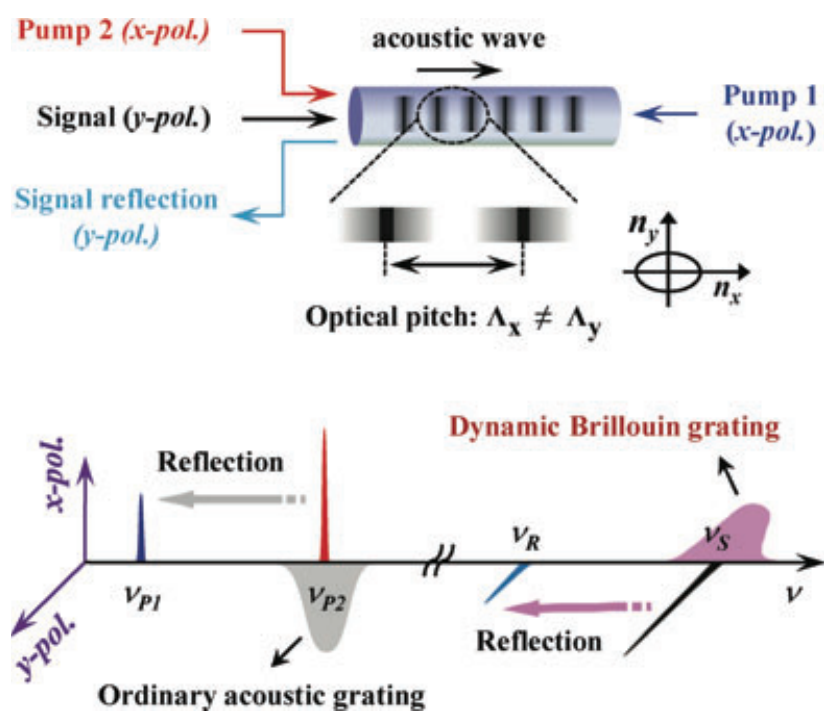

Figure 9 (online color at: www.lpr-journal.org) Graphical illustration of the principle for the generation of dynamic Brillouin grating reflectors in a polarization maintaining fiber, using the ordinary SBS process in a birefringent optical medium, where $v_{B}=v_{P 1}-v_{P 2}=v_{S}-v_{R}\left(v_{R}\right.$ being the frequency of the reflected light signal). 
which basically results from the essential frequency matching condition of the ordinary stimulated Brillouin scattering in fibers, given by $[60,61]$ :

$$
v_{B}=\frac{2 V_{a}}{c} n_{x} v_{P 1}^{x}=\frac{2 V_{a}}{c} n_{y} v_{S}^{y} .
$$

where $n_{x}$ and $n_{y}$ are the refractive indexes in the slow and fast axes of the fiber $\left(\Delta n=n_{x}-n_{y}\right)$, respectively, $\Delta n_{g}$ represents the group birefringence, by analogy calculated from $\Delta n$ using Eq.(1) and not so different from $\Delta n$ in standard fibers, and $V_{a}$ is the sound velocity. It can be clearly seen in Eq.(7) that, when the pumps propagate along the slow axis, the signal satisfies the Bragg condition at a frequency shifted above the pump frequency due to the simple fact that $n_{x}>n_{y}$. On the other hand, it can be straightforwardly deduced that when the polarization states of the pumps and signal are swapped, so that the grating is generated by two y-polarized pumps (fast axis), the signal frequency has to be down-shifted below the pump frequency, keeping the same spectral distance between Pump 1 and the signal.

\subsection{Principle of signal delaying for isolated pulses}

Before discussing the delaying principle itself, it is crucial to notice that the acoustic grating is present only where the SBS interaction takes place, hence where the two pump waves are simultaneously present and spatially overlap. It means that when the Brillouin pumps are shaped as optical pulses, the dynamic grating will be generated only at the particular position in the fiber where the two pulses are crossing.

Practically, as shown in Fig. 10, two optical pump pulses (Pump 1 and Pump 2) launched into a polarizationmaintaining fiber, each from a distinct end of the fiber, generate an acoustic grating at the position where the two pulses physically overlap. So, by properly delaying the Pump 1 pulse, the grating can be flexibly placed at any preset position over the entire fiber length. As a consequence, a signal pulse that enters into the fiber shortly after Pump 2 will be reflected by the grating before it decays. Basically, the tunability of the temporal delay is directly given by the timeof-flight change resulting from the grating repositioning,

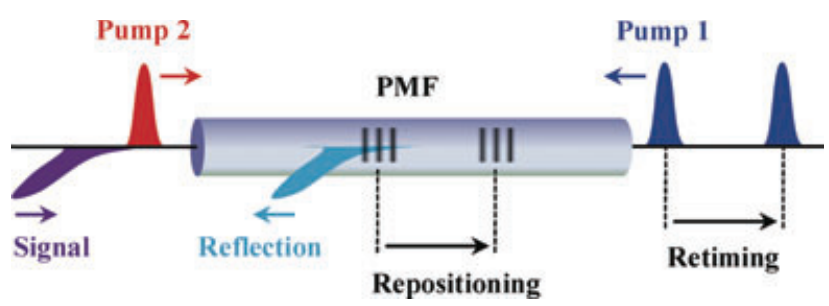

Figure 10 (online color at: www.lpr-journal.org) Principle to generate relative time delays through reflection of signal pulses in a polarization-maintaining fiber, realized by the optical generation of a Brillouin grating and dynamically moving the grating position. This results for the signal pulses in a varying round-trip time through the fiber. as shown in Fig. 10. This is physically equivalent to a bulk delay line made of a moveable mirror placed on a linear sliding scale, on which the light beam is reflected. In this case the delay change is twice larger than the equivalent reflector displacement. Therefore, it can be deduced by simple analogy that the maximal achievable time delay is determined by the length of the birefringent fiber and is equal to twice the transit time through the fiber.

\subsection{Experimental demonstration and discussion}

For the demonstration a $120 \mathrm{~m}$-long Panda-type polarization maintaining fiber was used as Brillouin gain. The birefringence $\Delta n$ of the fiber was experimentally measured to be $\Delta n \approx 5 \times 10^{-4}$, simply by measuring the differential Brillouin frequency shift between slow and fast axes. This way, Eq.(6) predicts that the DBG will be spectrally placed for the signal at about $46 \mathrm{GHz}$ above the Pump 1 frequency. From the same single-frequency laser diode Pump 1 and Pump 2 were shaped in the time domain as identical pulse trains of $600 \mathrm{ps}$ duration at a repetition rate of $2 \mathrm{MHz}$, with the exact frequency difference which maximizes the Brillouin interaction. The timing between the pulses can be independently changed with an electronic delay generator and the pulses power was boosted to several Watts to generate during the short overlapping time a grating intense enough to observe a few percent reflection [89].

A distinct single-frequency laser was used to generate the signal pulse that was shaped as an identical pulse train of $\sim 600$ ps duration and $2 \mathrm{MHz}$ repetition rate. The polarization state of the signal pulse was orthogonally aligned with respect to the two pumps. The central frequency of the signal pulse was precisely controlled by the current and temperature applied to the laser diode, for a perfect centering of the signal frequency on the middle of the grating resonance. Actually, the signal was spectrally placed at around $43 \mathrm{GHz}$ above the Pump 1 frequency for a maximum reflection, in excellent agreement with the prediction. The time interval between the signal and Pump 2 was set at 500 ps to minimize the decay of the grating amplitude that shows a typical time constant of $11 \mathrm{~ns}$. This way, the efficiency of the Bragg reflection on the signal pulse is nearly ideal. Figure 11 shows the measured intensity profiles and the time arrangement of the two pump and signal pulses used in this experiment.

To validate the feasibility of the realization of tunable time delays for the signal pulse, Pump 1 was independently delayed with respect to Pump 2 and signal, so that eventually Pump 1 enters the polarization-maintaining fiber with a different relative time. Then the temporal waveforms of the reflected signal were measured while continuously moving the grating position along the fiber by $100 \mathrm{ps}$ steps. Figure 12 shows selected time traces of the reflected pulses from the grating at four different grating positions. It is clearly observed that the back-reflected pulses from the grating are spaced by $200 \mathrm{ps}$, corresponding to the amount of change of the round-trip time. 


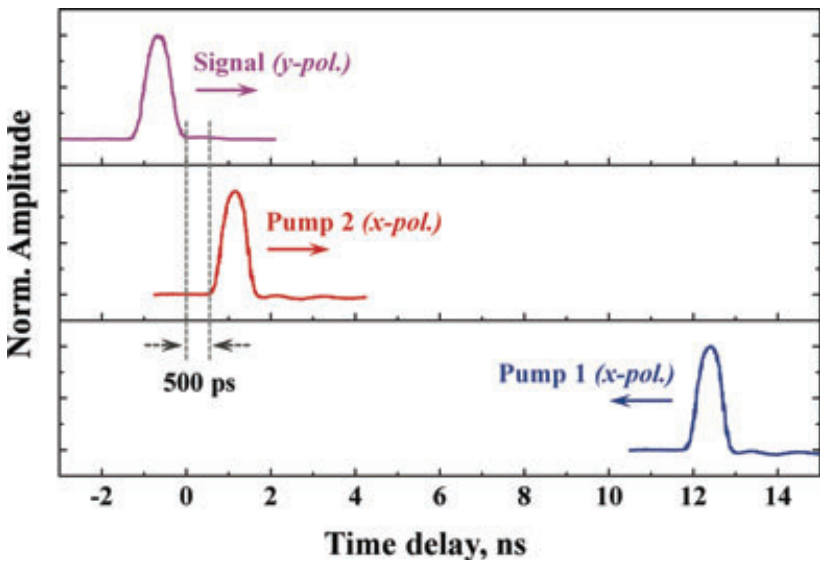

Figure 11 (online color at: www.lpr-journal.org) Configuration of pulse propagation of two pumps and signal through the fiber, showing the measured intensity profiles (nearly Gaussian shape) and time synchronization between the Pump 1 and signal with an interval of $500 \mathrm{ps}$.

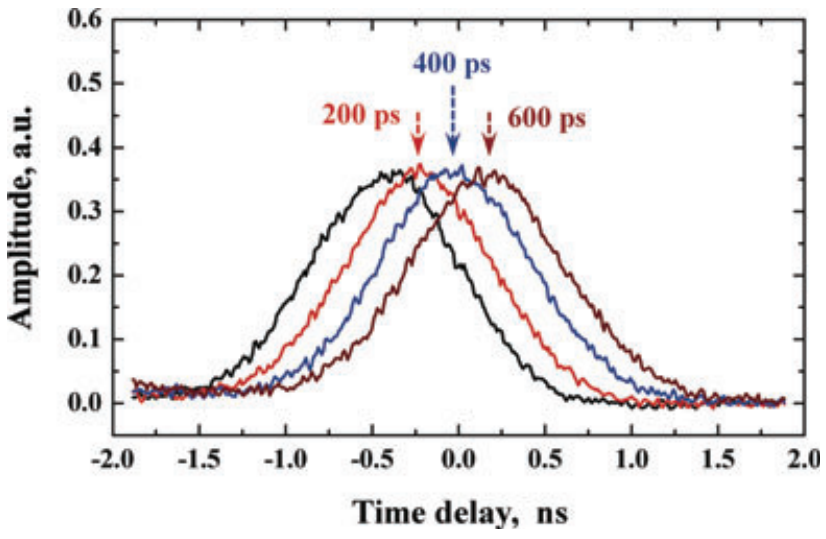

Figure 12 (online color at: www.lpr-journal.org) Temporal distribution of the reflected signal pulse from the dynamic Brillouin grating, localized at 4 different equally spaced positions (Pump 1 delay increment of $100 \mathrm{ps}$ ) showing a perfect pulse delaying.

In this type of dynamic reflectors, it can be assumed that the signal pulse linearly propagates through a weak fiber Bragg grating, and the perturbation due to the grating generates a reflected backward-propagating pulse. So the temporal shape of the reflected pulse is essentially determined by the convolution between the intensity profile of the original signal pulse and the temporal profile of the grating amplitude [90]. It means that the waveform of the reflected signal is influenced by the grating properties - essentially its physical length - and experiences some distortion. It can be reasonably assumed that the characteristics of the dynamic grating are unmodified for any positionning, since attenuation and dispersion are negligible over such short distances, so that the optical power and the temporal profile of Pump 1 and Pump 2 pulses are not position-dependent. For this reason, the response of the grating reflection is constant with respect to the grating position, and this was widely confirmed in practical implementations.

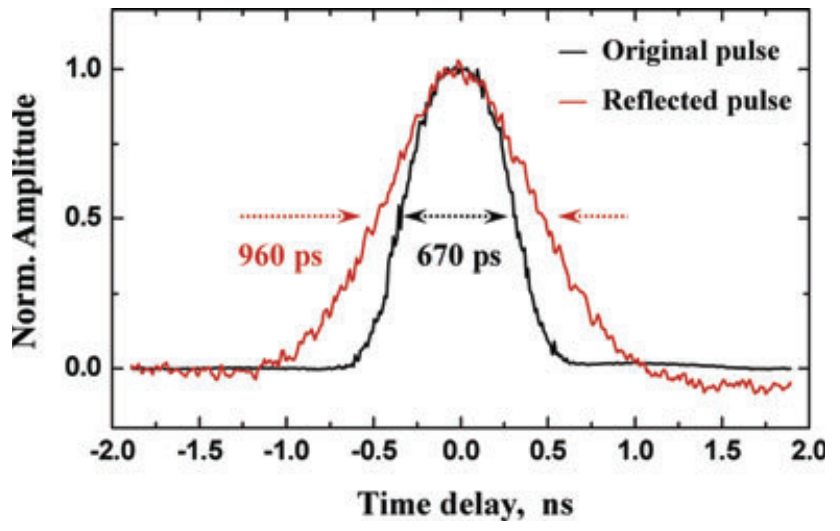

Figure 13 (online color at: www.lpr-journal.org) Comparison of the original signal pulse (in black) and the reflected pulse (in red) from the grating, clearly showing that the signal is broadened by a factor of 1.43 due to the fact that the relatively long grating continuously reflects the signal pulse as long as its physical length.

Since all interacting pulses have the same duration, the convolution makes the reflected pulse temporally broadened by a factor of 1.43 , when compared to the original pulse, as shown in Fig. 13. This pulse broadening can be visualized as the summation of the continuous reflection of the input signal pulse along the entire grating length. As a result of the gradual overlapping of the pump pulses, the temporal distribution of the grating is not uniform, but follows the time convolution between Pump 1 and Pump 2. It turns out that the length of the generated grating is comparable to the signal pulse spatial coverage and a reflected pulse broadened by a factor $\sqrt{2}$ is expected in this situation. This signal distortion can be minimized by simply shorten the pump pulses, but this will be done at the expense of the overall grating reflectance and a proper compensation of the pumps optical power is normally required to maintain the same level of reflectance $[60,61]$.

From a practical point of view, it was observed that the non-uniformity of the fiber birefringence can degrade the performance of this type of optical delay line. If the distribution of birefringence is inhomogeneous along the fiber, its fluctuation will lead to a spectral shift of the peak reflection resonance, as a direct consequence of the relation between birefringence and grating central frequency given by Eq.(6). Consequently, the frequency of the signal pulse may be locally detuned from the grating frequency and the efficiency of pulse reflection can therefore be significantly reduced, giving rise to a position-dependent reflected amplitude and a modified spectral distribution [91]. So this technique definitely requires the choice of a fiber with good birefringence uniformity for a proper operation.

To further demonstrate the feasibility and physical limitations of this technique, the grating was continuously shifted by $10 \mathrm{~ns}$ steps over the entire fiber length. In Fig. 14, superposed time traces of the reflected signal pulses for different grating positions are plotted, clearly showing a substantial delaying of the reflected pulse. The peak position of the reflected pulse was then used to determine the amount of time delay and the full set of possible delaying is 


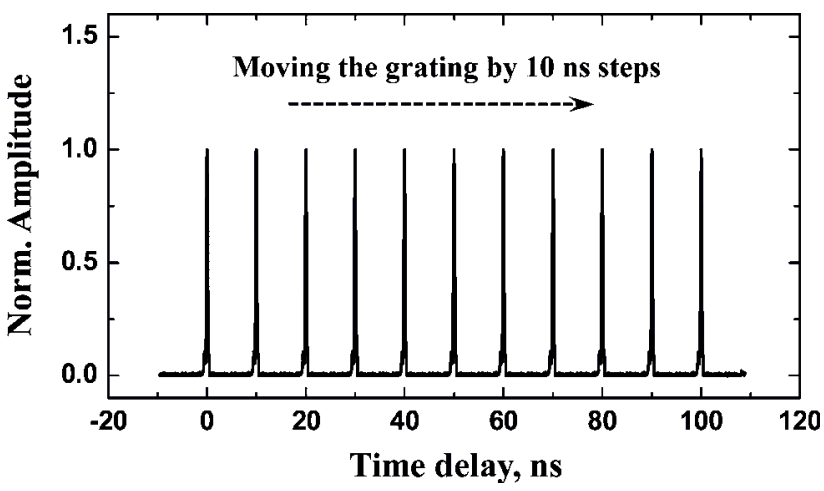

Figure 14 Normalized time traces of the delayed signal pulse while moving the grating position by 10 ns steps.

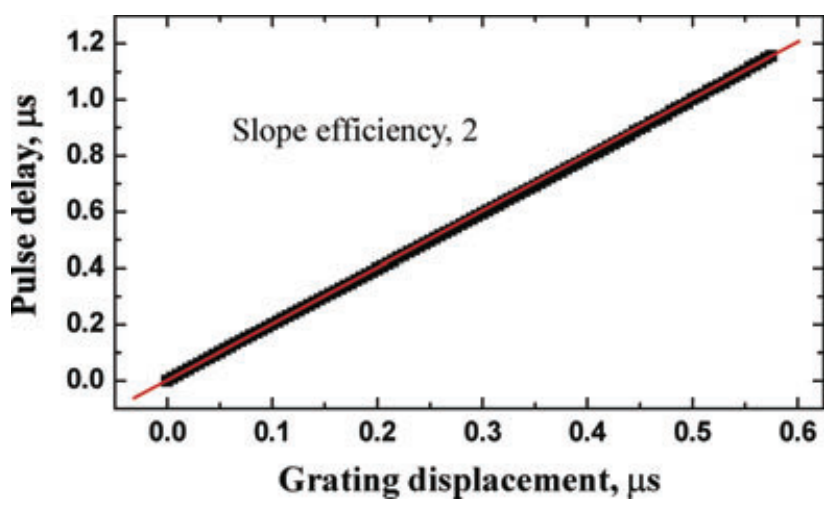

Figure 15 (online color at: www.lpr-journal.org) Time delays for the reflected pulse with respect to the grating displacement, showing ultra large continuous delays up to $1.15 \mu \mathrm{s}$, equivalent to a 1716-bits normalized delay.

shown in Fig. 15. It obviously confirms that the time delay of the back-reflected signal shows a linear dependence on the grating position with a conversion factor of 2 , as expected for a round-trip propagation. The largest time delay obtained using this delay line was $1.15 \mu \mathrm{s}$, corresponding to a normalized delay of 1716-bits for this $600 \mathrm{ps}$ pulse, basically limited by the available fiber length. Actually, the maximum achievable delay can be simply expanded by using a longer fiber. However, it must be mentioned that the intrinsic fiber properties such as nonlinearities, polarization crosstalk and birefringence walk-off limit the realistic dimension of the polarization maintaining fiber to length shorter than $1 \mathrm{~km}$, nevertheless corresponding to a potential continuous delaying up to $10 \mu$ s.

\subsection{Modified scheme for delaying continuous data streams}

The delay line presented in the previous section is, however, applicable for isolated pulses only, due to the fast decaying property of acoustic phonons. In general, the generated dynamic grating decays exponentially in time, and this effect makes the grating non-permanent. It is a serious penalty for

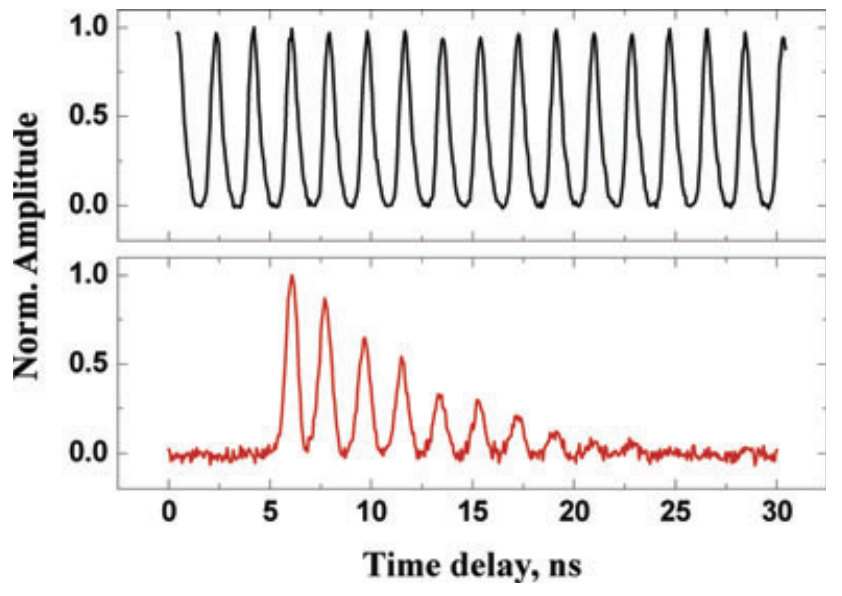

Figure 16 (online color at: www.lpr-journal.org) Time trace of the original (upper) and reflected (lower) signal pulse train by a dynamic grating generated by crossing short pulses. This illustrates the actual limitation of this delaying system in terms of the maximal data packet length resulting from the finite acoustic lifetime.

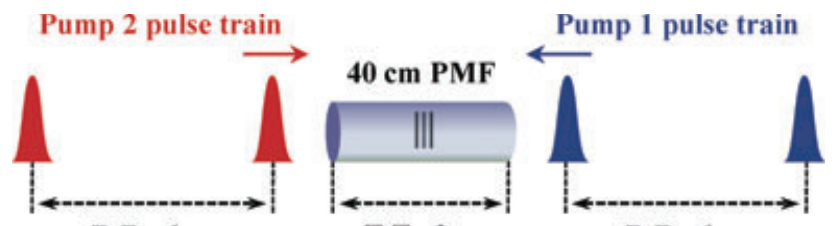

R.R. $6 \mathrm{~ns}$

T.T. 2 ns

R.R. 6 ns

Figure 17 (online color at: www.lpr-journal.org) Configuration of pump pulses to generate a quasi-permanent dynamic grating in a PM fiber, by periodically refreshing the grating using a sequence of pump pulses. R. R.; repetition rate, T. T.; transit time through the fiber.

this type of delaying system since it concretely limits the total length of the data packet carrying the information. Actually, the packet length cannot much exceed the acoustic lifetime $\tau_{B}$, (typically $11 \mathrm{~ns}$ in standard optical fibers), as illustrated in Fig. 16. In the test shown in this figure a $500 \mathrm{ps}$ pulse train at a repetition rate of $1.5 \mathrm{~ns}$ is reflected by a dynamic grating generated by isolated pump pulses crossing in a polarization-maintaining fiber. It is clearly observed that only a fraction of the pulse train is noticeably reflected as a consequence of the gradual vanishing of the grating amplitude, on a time scale given by the acoustic lifetime.

This lifetime $\tau_{B}$ depends on intrinsic parameters of the material such as medium viscosity and incident frequency of the pump wave, but remains of the same order of magnitude for all glass materials with potentialities in modern photonics. However, the dynamic grating can be periodically regenerated at a desired position, by simply using a sequence of pump pulses at a repetition rate faster than the acoustic lifetime, so that it can be made quasi-permanent and be kept localized in the fiber. A configuration for the generation of quasi-permanent dynamic gratings is sketched in Fig. 17. Actually, the repetition rate of Brillouin Pump 1 and Pump 2600 ps pulse trains is adjusted at $6 \mathrm{~ns}$, shorter 


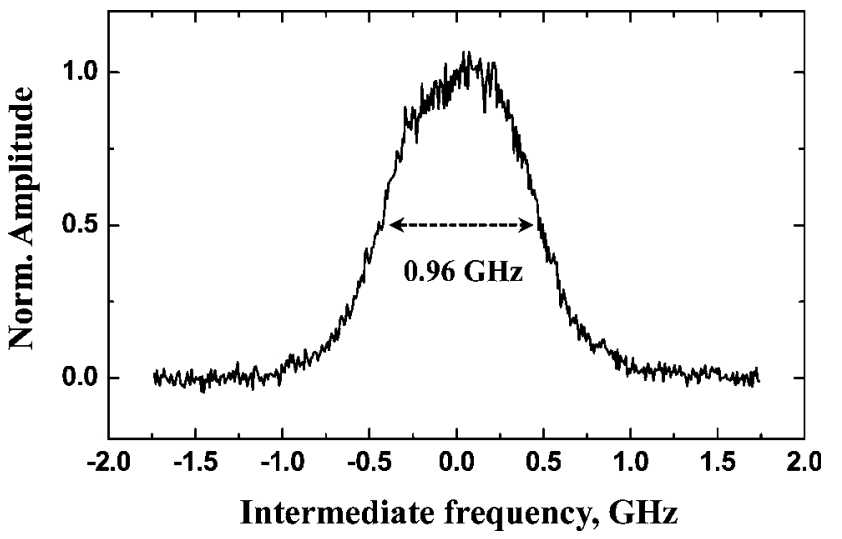

Figure 18 Variation of the amplitude of a reflected CW probe wave on a dynamic Brillouin grating as a function of the optical frequency. This represents the spectral response of the dynamic grating resonance, showing a FWHM spectral bandwidth of $0.96 \mathrm{GHz}$ that is primarily determined by the pump pulses duration. Frequency origin is arbitrarily placed at the grating center.

than the $11 \mathrm{~ns}$ acoustic lifetime. So, the dynamic grating is periodically refreshed at the same position before it vanishes. The length of the polarization-maintaining fiber used as delay line was also accordingly shortened to $40 \mathrm{~cm}$, corresponding to a $2 \mathrm{~ns}$ transit time. This way, only one dynamic grating is securely present along the entire $40 \mathrm{~cm}$ fiber, the condition being that the transit time must be less than the half repetition rate.

A set of characterization has been carried out, starting by the measurement of the reflective spectral properties of the generated dynamic grating. To spectrally analyze the grating reflectance, the frequency of a $\mathrm{CW}$ signal in the vicinity of the reflection band of the dynamic grating is swept. The grating is spectrally centered $43 \mathrm{GHz}$ above the Pump 2 frequency and the measured spectral dependence of the reflected light is shown in Fig. 18. The bandwidth of the grating is measured to be $0.96 \mathrm{GHz}$ at full width at half maximum, in close agreement with the $0.86 \mathrm{GHz}$ theoretical expectation $[82,92]$. As previously mentioned, the bandwidth that can be handled by this type of delay line is essentially determined by the spatial length of the grating and can be flexibly modified by simply adapting the duration of the pump pulses to the signal symbol duration.

To illustrate the capability to delay a temporally dense signal a $840 \mathrm{ps}$ pulse train at a repetition rate of $4.4 \mathrm{~ns}$ is generated with its frequency precisely centered on the grating resonance. Unlike the previous pulse delay experiment, a temporal synchronization between pump and signal pulses is no longer necessarily required since the dynamic grating is refreshed at a fixed position in the fiber and continuously reflects the signal. Figure 19 shows the normalized time waveforms of the reflected pulse trains while the grating is shifted along the fiber. The pulse train is clearly delayed without significant signal distortion for different grating positions. The largest time delay induced by the proposed scheme was $3.3 \mathrm{~ns}$, corresponding to a fractional delay of 4 . This ratio is basically limited up to 4.7 for 840 ps since the

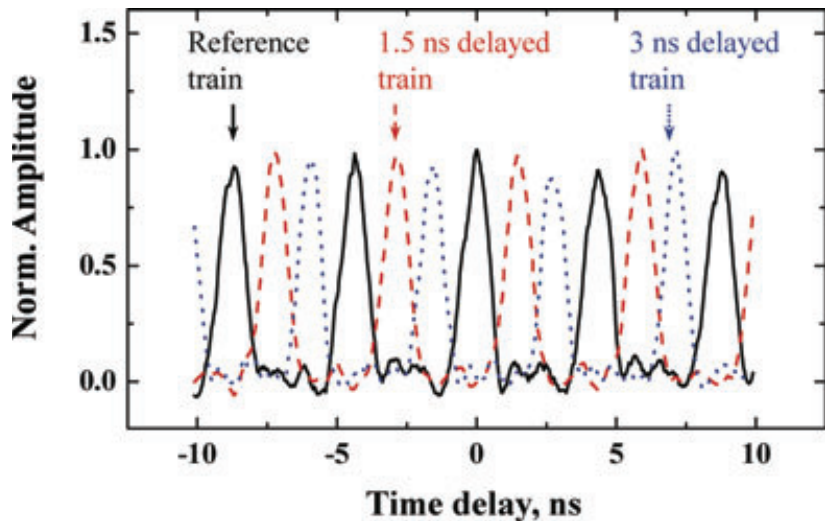

Figure 19 (online color at: www.lpr-journal.org) Time traces of delayed signal pulse trains while shifting the grating by $1.5 \mathrm{~ns}$ steps, clearly showing the possibility to realize tunable time delays for continuous data streams.

maximum achievable time delay is physically determined by the $40 \mathrm{~cm}$ fiber length.

Considering that the maximum refreshing time is of the order of the $11 \mathrm{~ns}$ acoustic lifetime in the fiber, this also corresponds to the maximum delay range that can be realized using this refreshing technique. This actually limits the normalized delay for a $10 \mathrm{Gbit} / \mathrm{s}$ data sequence $(100 \mathrm{ps}$ symbol duration) to a bit more than 100 . This is substantially better than what can be achieved using slow light, but not sufficient for an implementation in a digital transmission system. But promising solutions are currently investigated to generate permanent localized gratings at a fixed position, based on the correlation properties of random or chaotic pump signals to build an efficient acoustic grating at a single location over a very long fiber.

A clear unique asset of this technique is related to the fact that the transit time in the system ranges from 0 to the maximum delay, unlike the two other presented techniques that always show a nominal transit time normally substantially longer than the delay range that can be achieved. It means that the amount of delay can be reconfigured ideally rapidly, with a typical time equal to the delay range, which is the theoretical limit for all delay lines. However it must be mentioned that the delay is here first generated electrically by temporally ordering the emission time of the two optical pump pulses using an electrical delay generator. But, all in all, this is not fundamentally different to other techniques in which the amount of delaying is also originally function of an electrical signal, e. g. pump driving current in a slow light system or wavelength setting in a dispersive delay line.

\section{Conclusion}

We have analyzed three major currently available optical timing systems that can provide continuously tunable signal delaying for broadband optical signals: slow and fast light, wavelength conversion associated with a dispersive medium and dynamic grating reflectors. To date, we reach the temporary conclusion that all techniques still suffer from 
Table 1 Comparison of the performances and limitations of the 3 presented all-optical delaying systems. The figures shown are for typical implementations employing state-of-the-art devices and do not represent record cases.

\begin{tabular}{|c|c|c|c|}
\hline & Slow \& fast light & WC-based delay line & DBG-based delay line \\
\hline $\begin{array}{l}\text { Maximum achievable } \\
\text { time delay }\end{array}$ & $\begin{array}{l}1-2 \text { bits delay for isolated pulses } \\
\text { and real data streams }\end{array}$ & $\begin{array}{l}>1000 \text { bits for isolated pulses } \\
\text { and real data streams }\end{array}$ & $\begin{array}{l}>1000 \text { bits for isolated pulses } \\
<11 \mathrm{~ns} \text { absolute delay for data } \\
\text { streams }\end{array}$ \\
\hline Signal bandwidth & $\begin{array}{l}>10 \mathrm{GHz} \\
\text { The larger the signal bandwidth, } \\
\text { the stronger the pump power }\end{array}$ & $\begin{array}{l}>10 \mathrm{GHz} \\
\text { The larger the signal bandwidth, } \\
\text { the stronger the GVD broadening }\end{array}$ & $\begin{array}{l}>10 \mathrm{GHz} \\
\text { The larger the signal bandwidth, } \\
\text { the stronger the pump power }\end{array}$ \\
\hline $\begin{array}{l}\text { Associated signal } \\
\text { distortion }\end{array}$ & $\begin{array}{l}\text { The larger the time delay, the } \\
\text { stronger the pulse broadening }\end{array}$ & $\begin{array}{l}\text { The larger the time delay, the } \\
\text { stronger the pulse broadening }\end{array}$ & $\begin{array}{l}\text { Broadening factor independent of } \\
\text { delay, reduced by shorter pulses } \\
\text { and an increased pump power }\end{array}$ \\
\hline Reconfiguration time & $\begin{array}{l}>5 \mu \mathrm{s} \\
\text { Desired time delay } \\
+ \\
\text { Optical length of gain medium } \\
(\sim 1 \mathrm{~km} \text { fiber })\end{array}$ & $\begin{array}{l}>5 \mu \mathrm{s} \\
\text { Desired time delay } \\
+ \\
\text { Optical length of dispersive } \\
\text { medium }(\sim 1 \mathrm{~km} \text { fiber }) \\
+ \\
\text { Optical length of WC medium }\end{array}$ & $\approx$ Desired time delay \\
\hline
\end{tabular}

a common trade-off between the maximum achievable time delay and signal distortion. The ideal system that fulfills the requirements of the roadmap to all-optical digital delay lines for the future all-optical routers does not yet exist, but significant progresses were observed these past years and a solution will probably be found over a human timeline. The capabilities demonstrated today by each technique and their limitations are summarized in Table 1 for a better comparison. If the systems based on slow and fast light have been extensively studied and no significant progress is further expected, the two other techniques are still under intensive development and can yet substantially improve their performance. But, most importantly, these fascinating modern techniques have for the first time given access to an all-optical control of the temporal dimension and will provide more potential functionalities for all-optical signal processing, in communication systems, in microwave photonics and other advanced domains.

Acknowledgements. This work was supported by the Swiss National Science Foundation through project 200020-121860 and by the European Community's Seventh Framework Programme [FP7/2007-2013] under grant agreement No. 219299 (GOSPEL project).

Received: 15 September 2011, Revised: 5 December 2011, Accepted: 12 December 2011

Published online: 9 February 2012

Key words: Nonlinear fiber optics, stimulated Brillouin scattering, slow and fast light, optical signal processing, fiber Bragg grating.

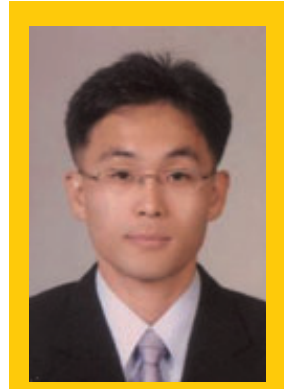

Sanghoon Chin received the M. Sc. degree in 2005 in information and communication from Gwangju Institute of Science and Technology (GIST), South Korea. In 2009, he received the Ph. D. degree in Electrical Engineering from the Ecole Polytechnique Fédérale de Lausanne (EPFL), Switzerland, where he developed his expertise on slow and fast light in optical fibers. His research has been mainly oriented to develop the dynamic control of the speed of a light signal in optical fibers, using stimulated Brillouin scattering. In 2010, he visited the Polytechnique University in Valencia, Spain, and Thales Research \& Technology, Palaiseau, France, and gained an expertise in microwave photonics. He has joined in 2011 the company Omnisens.

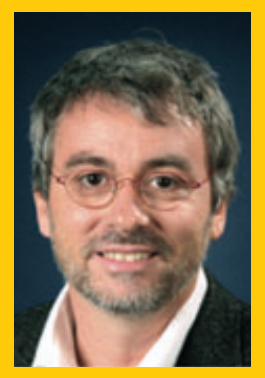

Luc Thévenaz received the M.Sc. degree in 1982 and the Ph. D. degree in physics in 1988, both from the University of Geneva, Switzerland. In 1988 he joined the Swiss Federal Institute of Technology of Lausanne (EPFL) where he currently leads a research group involved in photonics, namely fiber optics and optical sensing. Research topics include Brillouinscattering fiber sensors, slow and fast light, nonlinear fiber optics and laser spectroscopy in gases. He achieved with his collaborators the first experimental demonstration of optically controlled slow and fast light in optical fibers, and is at the origin of innovative configurations for Brillouin distributed fiber sensors, such as generation of the signal wave using modulation sidebands and Brillouin dynamic gratings. During his career he stayed at Stanford University, 
at the Korea Advanced Institute of Science and Technology (KAIST), at Tel Aviv University and at the University of Sydney. In 2000 he co-founded the company Omnisens that is developing and commercializing advanced photonic instrumentation. Prof. Thévenaz was Chairman of the European COST Action 299 "FIDES: Optical Fibres for New Challenges Facing the Information Society", has edited the book entitled "Advanced Fiber Optics" and is author or coauthor of some 350 publications and 7 patents. He is Fellow of the Optical Society of America.

\section{References}

[1] R. W. Boyd and D. J. Gauthier, Slow and Fast Light (Elsevier, Amsterdam, 2002), pp. 497-530.

[2] J. B. Khurgin and R. S. Tucker, Slow Light: Science and Applications (CRS Press Boca Raton, 2009).

[3] J. Capmany and D. Novak, Nature Photonics 1, 319-330 (2007).

[4] J. Yao, Nature Photonics 4, 79-80 (2010).

[5] E. Choi, J. H. Na, S. Rhu, G. Mudhana, and B. H. Lee, Opt. Express 13, 1334-1345 (2005).

[6] L. V. Hau, S. E. Harris, Z. Dutton, and C. H. Behroozi, Nature 397, 594-598 (1999).

[7] M. M. Kash, V. A. Sautenkov, A. S. Zibrov, L. Hollberg, G. R. Welch, M.D. Lukin, Y. Rostovtsev, E. S. Fry, and M. O. Scully, Phys. Rev. Lett. 82, 5229-5232 (1999).

[8] L. J. Wang, A. Kuzmich, and A. Dogariu, Nature 406, $277-$ 279 (2000).

[9] C. Liu, Z. Dutton, C. H. Behroozi, and L. V. Hau, Nature 409, 490-493 (2001).

[10] A. V. Turukhin, V.S. Sudarshanam, M.S. Shahriar, J. A. Musser, B. S. Ham, and P. R. Hemmer, Phys. Rev. Lett. 88, 023602 (2002).

[11] M.S. Bigelow, N. N. Lepeshkin, and R. W. Boyd, Science 301, 200-202 (2003).

[12] M. S. Bigelow, N. N. Lepeshkin, and R. W. Boyd, Phys. Rev. Lett. 90, 113903 (2003).

[13] P. C. Ku, F. Sedgwick, C. J. Chang-Hasnain, P. Palinginis, T. Li, H. Wang, S. W. Chang, and S. L. Chuang, Opt. Lett. 29, 2291-2293 (2004).

[14] J. Mork, R. Kjaer, M. Van der Poel, and K. Yvind, Opt. Express 13, 8136-8145 (2005).

[15] P. Palinginis, F. Sedgwick, S. Crankshaw, M. Moewe, and C. Chang-Hasnain, Opt. Express 13, 9909-9915 (2005).

[16] P. Palinginis, S. Crankshaw, F. Sedgwick, E. T. Kim, M. Moewe, C.J. Chang-Hasnain, H.L. Wang, and S. L. Chuang, Appl. Phys. Lett. 87, 171102 (2005).

[17] R. S. Tucker, P. C. Ku, and C. J. Chang-Hasnain, J. Lightwave Technol. 23, 4046-4066 (2005).

[18] F. Ohman, K. Yvind, and J. Mork, Opt. Express 14, 99559962 (2006).

[19] H. Su and S. L. Chuang, Appl. Phys. Lett. 88, 061102 (2006).

[20] Y. A. Vlasov, M. O'Boyle, H. F. Hamann, and S. J. McNab, Nature 438, 65-69 (2005).

[21] M. Notomi, K. Yamada, A. Shinya, J. Takahashi, C. Takahashi, and I. Yokohama, Phys. Rev. Lett. 87, 253902 (2001).

[22] T. F. Krauss, J. Phy. D Appl. Phys. 40, 2666-2670 (2007).

[23] D. Mori, S. Kubo, H. Sasaki, and T. Baba, Opt. Express 15, 5264-5270 (2007).
[24] J. E. Heebner and R. W. Boyd, J. Mod. Opt. 49, 2629-2636 (2002).

[25] J. K. S. Poon, J. Scheuer, Y. Xu, and A. Yariv, J. Opt. Soc. Am. B 21, 1665-1673 (2004).

[26] J. K. S. Poon, L. Zhu, G. A. DeRose, and A. A. Y. Y. Xu, Opt. Lett. 31, 456-458 (2006).

[27] Q. Xu, J. Shakya, and M. Lipson, Opt. Express 14, 64636468 (2006).

[28] J. B. Khurgin, Physical Review A 62, 013821 (2000).

[29] S. Longhi, D. Janner, G. Galzerano, G. Della Valle, D. Gatti, and P. Laporta, Electronics Letters 41, 1075-1077 (2005).

[30] Y. Dai, Y. Okawachi, A.C. Turer-Foster, M. Lipson, A. L. Gaeta, and C. Xu, Opt. Express 18, 333-339 (2010).

[31] S. Chin, M. Gonzalez-Herraez, and L. Thévenaz, Opt. Express 17, 21910-21917 (2009).

[32] L. Thevenaz and S. Chin, Comptes Rendus Physique 10, 1008-1013 (2009).

[33] Y. Okawachi, M. A. Foster, X. Chen, A. C. Turner-Foster, R. Salem, M. Lipson, C. Xu, and A. L. Gaeta, Opt. Express 16, 10349-10357 (2008).

[34] V. P. Kalosha, W. Li, F. Wang, L. Chen, and X. Bao, Opt. Lett. 33, 2848-2850 (2008).

[35] Z. Zhu, D. J. Gauthier, and R. W. Boyd, Science 318, 17481750 (2007).

[36] S. Chin, M. Gonzalez-Herráez, and L. Thévenaz, Opt. Express 14, 10684-10692 (2006).

[37] M. Gonzalez-Herráez, K. Y. Song, and L. Thévenaz, Opt. Express 14, 1395-1400 (2006).

[38] M. Fok and C. Shu, in: Proceedings of the ECOC'06, Cannes, France, 2006.

[39] G. M. Gehring, A. Schweisberg, C. Barsi, N. Kostinski, and R. W. Boyd, Science 312, 895-897 (2006).

[40] E. Shumakher, A. Willinger, R. Blit, D. Dahan, and G. Einstein, Opt. Express 14, 8540-8545 (2006).

[41] A. Schweinsberg, N. N. Lepeshkin, M. S. Bigelow, R. W. Boyd, and S. Jarabo, Europhys. Lett. 73, 218-224 (2006).

[42] Y. Okawachi, M.S. Bigelow, J.E. Sharping, Z. Zhu, A. Schweinsberg, D. J. Gauthier, R. W. Boyd, and A. L. Gaeta, Phys. Rev. Lett. 94, 153902 (2005).

[43] M. Gonzalez Herráez, K. Y. Song, and L. Thévenaz, Appl. Phys. Lett. 87, 081113 (2005).

[44] K. Y. Song, M. Gonzalez-Herráez, and L. Thévenaz, Opt. Express 13, 82-88 (2005).

[45] J.E. Sharping, Y. Okawachi, J. V. Howe, C. Xu, Y. Wang, A. E. Willner, and A. L. Gaeta, Opt. Express 20, 7872-7877 (2005).

[46] J.E. Sharping, Y. Okawachi, and A. L. Gaeta, Opt. Express 13, 6092-6098 (2005).

[47] A. Yeniay, J.-M. Delavaux, and J. Toulouse, J. Lightwave Technol. 20, 1425-1432 (2002).

[48] L. Thévenaz, Nature Photonics 2, 474-481 (2008).

[49] M. P. Fok and C. Shu, J. Lightwave Technol. 26, 499-504 (2008).

[50] Y. Okawachi, J. E. Sharping, C. Xu, and A. L. Gaeta, Opt. Express 14, 12022-12027 (2006).

[51] R. W. Boyd, D. J. Gauthier, A. L. Gaeta, and A. E. Willner, Phys. Rev., A 71, 023801 (2005).

[52] J. B. Khurgin, Opt. Lett. 32, 163-165 (2007).

[53] B. Zhang, L. S. Yang, J. Y. Yang, I. Fazal, and A. E. Willner, Photon. Technol. Lett. 19, 1081-1083 (2007).

[54] B. Zhang, L. Zhang, L. S. Yan, I. Fazal, J. Y. Yang, and A. E. Willner, Optics Express 15, 8317-8322 (2007). 
[55] B. Zhang, L. Yan, L. Zhang, and A. E. Willner, J. Lightwave Technol. 25, 3763-3769 (2008).

[56] P. A. Morton and J. B. Khurgin, Photon. Technol. Lett. 21, 1686-1688 (2009).

[57] J. Sancho, S. Chin, M. Sagues, A. Loayssa, J. Lloret, I. Gasulla, S. Sales, L. Thevenaz, and J. Capmany, Photon. Technol. Lett. 22, 1753-1755 (2010).

[58] S. Chin, J. Sancho, P. Berger, J. Bourderionnet, D. Dolfi, S. Sales, J. Capmany, and L. Thévenaz, Opt. Express 18, 22599-22613 (2010).

[59] H. Shin, A. Schweinsberg, C. Barsi, N. Kostinski, H. J. Chang, R. W. Boyd, Q.-H. Park, and D. J. Gauthier, Opt. Lett. 32, 906-908 (2007).

[60] R. W. Boyd, Nonlinear Optics, 2nd ed. (Academic Press, New York, 2003).

[61] G. P. Agrawal, Nonlinear Fiber Optics, 2nd ed. (Academic Press, San Diego, 1995).

[62] Z. Zhu, D. J. Gauthier, Y. Okawachi, J.E. Sharping, A. L. Gaeta, R. W. Boyd, and A. E. Willner, J. Opt. Soc. Am. B 22, 2378-2384 (2005).

[63] M. Gonzalez-Herraez and L. Thévenaz, Optics Express 17, 4732-4739 (2009).

[64] Y. Aoki and K. Tajima, J. Opt. Soc. Am. B 5, 358-363 (1988).

[65] G. C. Valley, J. Quantum Electron. QE-22, 704-712 (1986).

[66] M. Denariez and G. Bret, Phys. Rev. 171, 160-171 (1968).

[67] Z. Zhu, A. M. C. Dawes, L. Zhang, A.E. Willner, and D. J. Gauthier, J. Lightwave Technol. 25, 201-206 (2007).

[68] K. Y. Song and K. Hotate, Opt. Lett. 32, 217-219 (2007).

[69] S. Chin, Ph.D. Thesis No.4459, Ecole Polytechnique Fédérale de Lausanne, Lausanne, Switzerland (2009).

[70] R. Pant, M. D. Stenner, M. A. Neifeld, and D. J. Gauthier, Opt. Express 16, 2764-2777 (2008).

[71] S. Chin, M. G. Herraez, and L. Thevenaz, Opt. Express 15, 10814-10821 (2007).

[72] A. Zadok, O. Raz, A. Eyal, and M. Tur, Photon. Technol. Lett. 19, 462-464 (2007).

[73] S. Sandhu, M. L. Povinelli, M. F. Yanik, and S. Fan, Opt. Lett. 31, 1985-1987 (2006).

[74] J. T. Mok, C. M. Sterke, I. C. M. Littler, and B. J. Eggleton, Nature 2, 775-780 (2006).
[75] M. D. Stenner, M. A. Neifeld, Z. Zhu, A. M. C. Dawes, and D. J. Gauthier, Opt. Express 13, 9995-10002 (2005).

[76] D. A. B. Miller, Phys. Rev. Lett. 99, 203903 (2007).

[77] J. B. Khurgin, J. Opt. Soc. Am. B 22, 1062-1074 (2005).

[78] A. Wiatrek, R. Henker, S. Preußler, M. J. Ammann, A. T. Schwarzbacher, and T. Schneider, Opt. Express 17, 797802 (2009).

[79] M. Y. Frankel and R. D. Esman, IEEE Trans. Microw. Theory Techn. 43, 2387-2394 (1995).

[80] Y. Wang, C. Yu, L. Yan, A. E. Willner, R. Roussev, C. Langrock, M. M. Fejer, J. E. Sharping, and A. L. Gaeta, Photon. Technol. Lett. 19, 861-863 (2007).

[81] K. Y. Song, W. Zou, Z. He, and K. Hotate, Opt. Lett. 33, 926-928 (2008).

[82] K. Y. Song and H. J. Yoon, Opt. Lett. 17, 2958-2960 (2010).

[83] W. Zou, Z. He, and K. Hotate, Opt. Express 17, 1248-1255 (2009).

[84] Y. Dong, X. Bao, and L. Chen, Opt. Express 35, 193-195 (2010).

[85] K. Y. Song, S. Chin, N. Primerov, and L. Thévenaz, J. Lightwave Technol. 28, 2062-2067 (2010).

[86] K. Y. Song, K. Lee, and S. B. Lee, Opt. Express 17, 1034410349 (2009).

[87] N. Primerov, S. Chin, K. Y. Song, and L. Thevenaz, in: Optical Fiber Communication Conference, OSA Technical Digest (CD), San Diego, USA, 2010, paper OWF6.

[88] S. Chin, N. Primerov, S. Sales, and L. Thévenaz, in: CLEO/Europe and EQEC 2011 Conference Digest, OSA Technical Digest (CD), Munich, Germany, 2011, paper CD_P8.

[89] M. Nikles, L. Thevenaz, and P. A. Robert, J. Lightwave Technol. 15, 1842-1851 (1997).

[90] L. R. Chen, S. D. Benjamin, P. W. E. Smith, and J. E. Sipe, J. Lightwave Technol. 15, 1503-1512 (1997).

[91] Y. Park, M. Kulishov, R. Slavík, and J. Azaña, Opt. Express 14, 12670-12678 (2006).

[92] T. Erdogan, J. Lightwave Technol. 15, 1277-1294 (1997). 